\title{
Design of SmartMoving, an application for pedestrians with reduced mobility
}

\author{
Mónica Fatecha, Patricia Fauvety, Nathalie Aquino, Magalí González, \\ Daniel Romero, Luca Cernuzzi \\ Universidad Católica "Nuestra Señora de la Asunción" \\ Asunción, Paraguay \\ \{monica.fatecha, nathalie.aquino, mgonzalez, lcernuzz\}@uc.edu.py, \\ \{patyfauvety, danielomarromero\}@gmail.com
}

and

\author{
Javier Paniagua, Ronald Chenú-Abente \\ University of Trento \\ Trento, Italy \\ jpaniagualaconich@gmail.com, chenu@disi.unitn.it
}

\begin{abstract}
The state of sidewalks in Asunción, Paraguay is far from being optimal. There are many problems such as obstructions, disrepair, lack of ramps, surface unevenness, among others. In addition, the Municipality of Asunción does not have automated mechanisms to assess the state of sidewalks. In this work we propose SmartMoving, a mobile application that collects information on the state of sidewalks, with the help of citizens, and recommends pedestrian paths with fewer obstacles. The application can be especially useful for people with reduced mobility, as well as for the Municipality of Asunción. Due to their heavy reliance on citizen participation for data gathering, applications like SmartMoving require a particularly friendly user experience that is adapted to users and their daily context. In this cases, participatory and user-centered design methodologies are well-suited for application development. Therefore, in this paper we present the SmartMoving application and the user-centered design process that has been followed for its development, involving users with reduced mobility.
\end{abstract}

Keywords: pedestrian mobility, reduced mobility, citizen participation, user-centered design

\section{Introduction and motivation}

In recent years, several countries have experienced an increasing awareness of the importance of citizen participation as a primary factor in the development of society. Citizen participation has been shown to strengthen the links between citizens and public institutions, in order to guarantee the fulfillment of citizen rights [1]. Innovative solutions to problems of public interest can be made possible by providing technologies that create spaces of participation where citizens and government institutions can collaborate together. However, the availability of technological means is not enough in itself to achieve the general involvement of the population, it is also required to motivate citizens to use these technologies. In this sense, previous research has shown that citizens feel motivated to participate in decisions and discussions when these relate to their daily environment and are aimed at improving their quality of life [2].

The poor condition of the sidewalks is a key problem in the daily life of citizens in Asunción, Paraguay, and especially so for citizens with reduced mobility. When circulating on the streets, citizens find deteriorated sidewalks, commercial stalls located in prohibited places, waste, or construction sites that do not meet the requirements for free and safe pedestrian traffic. Due to these obstacles, it is often impossible for pedestrians 
to walk on the sidewalks, having to do it on the roadway, despite the danger that this represents for both pedestrians and drivers.

According to data we obtained in an 2018 interview with staff at the Municipality of Asunción, there were a total of 1,836 complaints related to problems in the sidewalks (749 due to obstruction and occupation, 686 related to construction and repair, 213 due to lack of ramps and 188 about slopes). However, the Municipality does not have the technological tools that would allow a continuous monitoring and management of the situation. Providing these tools would be very valuable for the task of getting complete and up-to-date information about the state of the sidewalks.

According to data from a different survey carried out in Paraguay in 2017, $94 \%$ of those surveyed have a smartphone and $86 \%$ have it connected to the Internet 3. Taking advantage of this situation, this work proposes that citizens use their smartphones connected to the Internet to collectively build and update information about the state of the sidewalks. This information collected by the citizens in a participatory manner, will be shared back with the citizens, which could help to improve pedestrian mobility in the city of Asunción. To this end, we present a mobile application called SmartMoving. It is worth mentioning that SmartMoving does not aim to repair the sidewalks themselves, but rather aims at helping pedestrians use these sidewalks, providing information on alternative pedestrian paths with fewer obstacles.

Since SmartMoving is based on user participation, it is important to make the application intuitive and useful. For this reason, the development of the application has been carried out following a User-Centered Design methodology [4] (UCD). Furthermore, considering that SmartMoving can be particularly useful for people who have reduce mobility, the process of design and evaluation of the interaction was carried out with the participation and collaboration of people that belong to that group. They participated in three iterations of analysis, design and evaluation, collaborating through interviews and a usability test.

It should be noted that this work deals with a problem installed in the city of Asunción and proposes a technological solution that has the potential to alleviate the problem of pedestrian circulation for people with reduced mobility. Even though the proposal will not be able to make Asunción a more inclusive city, it will be able to help people with reduced mobility so they are better informed about the condition of the sidewalks and more easily move around.

This paper is an extended version of [5]. It adds a new section (Section 3) that describes the SmartMoving application, its main features, architecture and implementation strategies. Part of this information was previously presented in [6] and now more details have been incorporated, including a description of the client and server architecture as well as discussions about information reliability and the routing algorithm. In addition, in this work we strengthen the qualitative analysis of the interviews carried out in the UCD process, incorporating a discussion about some relevant comments and suggestions from participants (see sections 4.3 and 4.4. Finally, a new section describes the findings and challenges for both the design process and the usefulness and potential adoption of applications like SmartMoving (see section 5).

The rest of this paper is structured as follows: section 2 presents related work; in section 3 functionalities, architecture and implementation strategies of SmartMoving are presented; section 4 presents the SmartMoving interaction layer design process, including the process planning and the three iterations performed; section 5 presents findings and discussion; and finally, section 6 presents conclusion and future work.

\section{Related work}

This section describes existing literature that tackles the generic problem of automatic route planning and also the more specific variants that deal with accessibility-related scenarios.

$m P A S S$ [7, 8] is a prototype system for personalized accessible routes. It provides three types of report: sensor, user and authority. In the first design iteration a low-fidelity prototype was evaluated by a group of 60 people with disabilities. They were presented with a questionnaire and mockups were used to walk them through. At the end of this first iteration a working prototype was implemented. In the second iteration this working prototype was evaluated by a group of 10 users. The evaluation focused on the route planning functionality and it was performed using the thinking aloud method. This project presents several interesting features and a sound evaluation strategy. Nevertheless, the type of incidents that users can report is different from those that SmartMoving focuses on, which align to the local context of the city of Asunción.

CrowdWatch 9] helps distracted pedestrians alerting them of transient obstacles (e.g., parked vehicles, garbage) automatically by means of GPS, accelerometer and compass sensors present in smartphone devices. It works by recommending the user to switch sidewalks in order to avoid these obstacles. When an incident is first reported, the interaction is kept simple, focusing on the location of the incident. When a second user walks by the same location, they are asked to confirm the first report by taking a photo of the incident. Although an evaluation was performed with 30 users, it was focused on the interface provided for each functionality rather than on the behavior and impact on the users. 
ParticipAct [10] is an application that displays a map of the city with points of interest and obstacles to mobility (e.g., stairs, scaffolding). An evaluation was performed but it was aimed at measuring memory and CPU usage in user devices.

Additionally, we explored the landscape of publicly available mobile applications similar to SmartMoving on the iPhone and Android application repositories. We downloaded, installed and attempted to test these applications, aiming to try their incident reporting and route planning functionalities. In some cases the applications promptly notified us that they were not available in our location. For the rest, even though the application would run, no users and lack of data about sidewalks meant that no test could be performed. These mobile applications are described below.

CIT2ADM For Pedestrians 17. users of this application can report any kind of incident found while they are using the sidewalk (e.g., lack of public lights or crosswalks, obstructions). It was designed to help municipalities, simplifying reporting and enabling real-time gathering of incidents. Its availability in a given location is subject to a contract between the application provider and the corresponding municipality.

Accessibility Plus ${ }^{2}$ lets users query for points of interest that have accessible amenities such as bathrooms, parking and others. Users can report new amenities, problems with existing ones and plan trips that avoid points of interest that are not accessible. The application is not available in our location.

GoogleMaps ${ }^{3}$ is a general purpose online mapping service. Although accessibility awareness is implemented for wheelchair users in the form of route planning that avoids obstacles, this feature is available in select locations only.

WheelMap ${ }^{4}$ displays a map with locations and their accessible amenities. Users can contribute with their reports to keep the database updated.

Yoya 5 is an application developed in Paraguay to evaluate locations regarding their accessibility for people with disabilities. The application is no longer available for download.

All the applications reviewed include some form of crowd sourcing either through explicit contributions by means of direct user input or through automatic sampling of sensors present in smartphone devices. This crowd sourcing activity keeps relevant information up-to-date and is a potential source of information that can be used by municipal authorities to guide city maintenance and development and by organizations that promote accessibility.

SmartMoving aims at filling the gap in application availability in Paraguay, providing a map to display the state of sidewalks and route planning that takes that information into account to implement accessibility awareness. Additionally, its design and implementation follows a user-centered approach that takes continuous feedback from its intended audience at all stages.

\section{The SmartMoving application}

In this section we describe the SmartMoving application [6] with a focus on main features, architecture design and implementation strategies.

\subsection{Features}

SmartMoving presents users with a map of their surroundings, representing the state of sidewalks and recommending routes that avoid inaccessible points. To model the state of sidewalks it overlays map information with user reports of incidents that affect their accessibility.

In this context, the main features of the application are:

- Visualization of the surroundings with focus on street and sidewalk geometry and incidents.

- Incident report creation and update.

- Report confirmation and resolution.

- Per-user configuration of incident severity by type.

- User-tailored route planning.

1 https://www.cit2adm.com/p/cit2adm-for-pedestrians

${ }^{2}$ https://play.google.com/store/apps/details?id=es.sdos.accesibility\&hl=en

3 https://play.google.com/store/apps/details?id=com.google.android.apps.maps\&hl=en

${ }^{4}$ https://play.google.com/store/apps/details?id=org. wheelmap.android.online\&hl=en

5 https://es-es.facebook.com/pg/yoyapy/community/ 


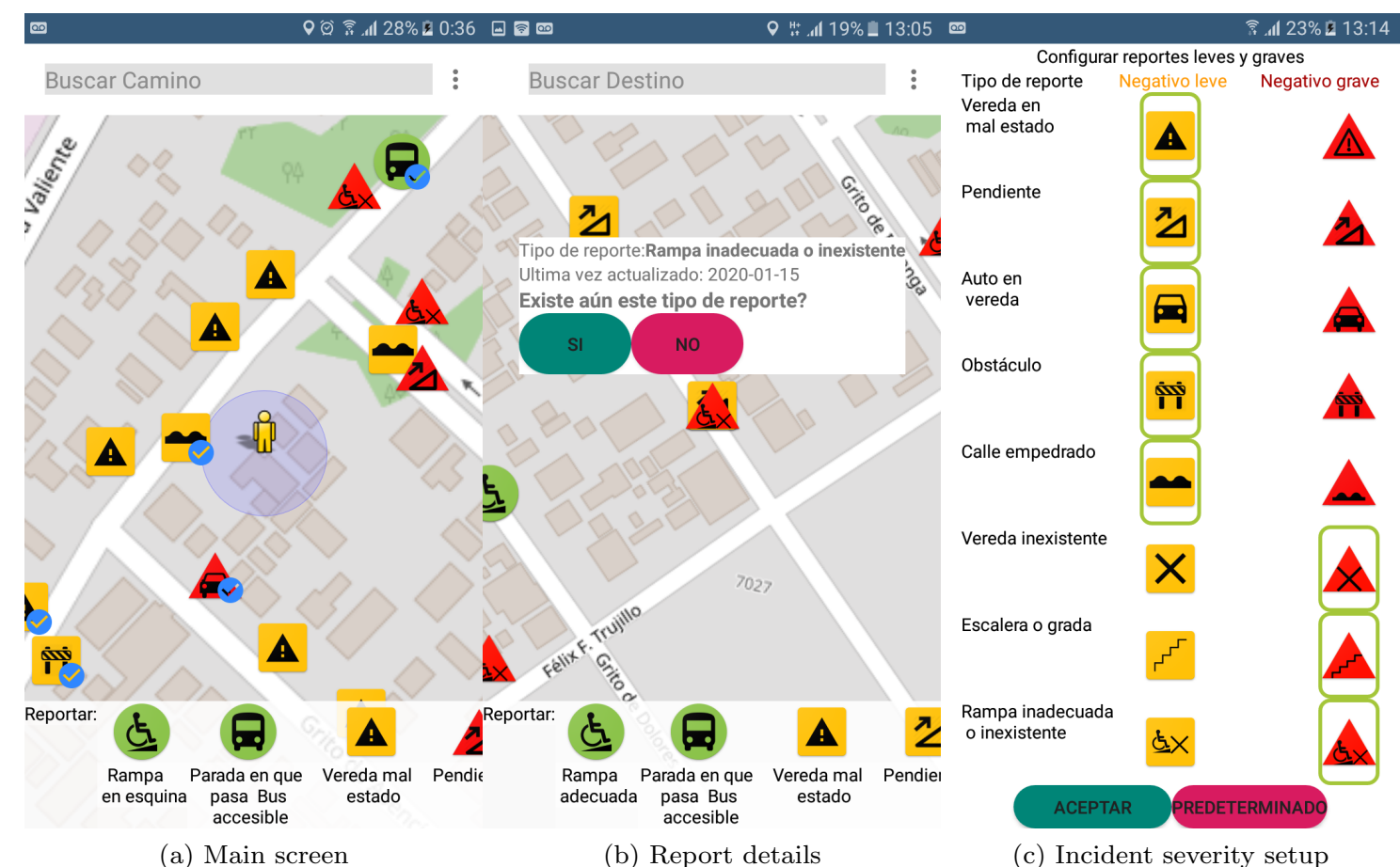

Figure 1: POI reports in SmartMoving

Figure 1 presents screen captures of the SmartMoving application with activities related to POI reports. Figure 1 1 shows the main screen with a map of the surroundings, including the location of the user and current nearby POI (Point of Interest) reports that were created by other users. These POI reports describe either situations that could present accessibility problems (e.g., lack of accessible street crossings, degraded or non-existent sidewalk, stairs, sidewalk obstruction by vehicle, uneven pavement) or the availability of accessible amenities (e.g., street crossing with ramp, stop for accessible bus). These POI reports are represented by markers in different colors and shapes, following the metaphor of traffic lights and signs: green circular markers represent usable accessible features, triangular markers represent accessibility problems with yellow and red colors representing minor and major problems respectively.

Users can create new POI reports touching the available icons in the bar at the bottom of the screen as seen in Figure 1 $1 \mathrm{a}$ and Figure 1 b and then touching the point in the map that better represent the location of the new report. In this way, a new report is available for all users in the vicinity.

The state of existing POI reports can be inspected by touching the corresponding icon on the map. As seen in Figure 1 1 , a brief detail prompt will pop up offering two actions to either confirm that the incident reported is still ongoing or to mark its resolution (e.g., because the vehicle that was obstructing the sidewalk has been removed).

Figure 1 1 shows the Incident Severity Setup screen, in which users can further describe how negative POI reports should be scored by the automatic route planner. In this way, users can tailor the behavior of the algorithm to better suit their needs.

Figure 2 shows a sequence of screen captures related to the automatic route planner. If the user touches the input control labeled "Find Path" on the top part of the screen in Figure 2 a, the desired destination can be input as a street address, assisted by auto-completion, as seen in Figure 2 p. Once a destination is selected, it is shown with a red marker as seen in Figure 2. The user can fine-tune the destination by dragging the map before starting the navigation activity. As seen in Figure 2 $\mathrm{d}$, once the user starts the navigation activity the recommended route is displayed on the map alongside the destination, the current position of the user, and all nearby POI reports. During the navigation activity the user can contribute new POI reports and mark existing reports as ongoing or resolved. As seen in Figure 2p, once the destination is reached the application notifies the user and ask for an evaluation of the automatic recommendation using a 1 to 5 stars rating scale. The flow will go back to Figure 2 a after the evaluation is provided. 


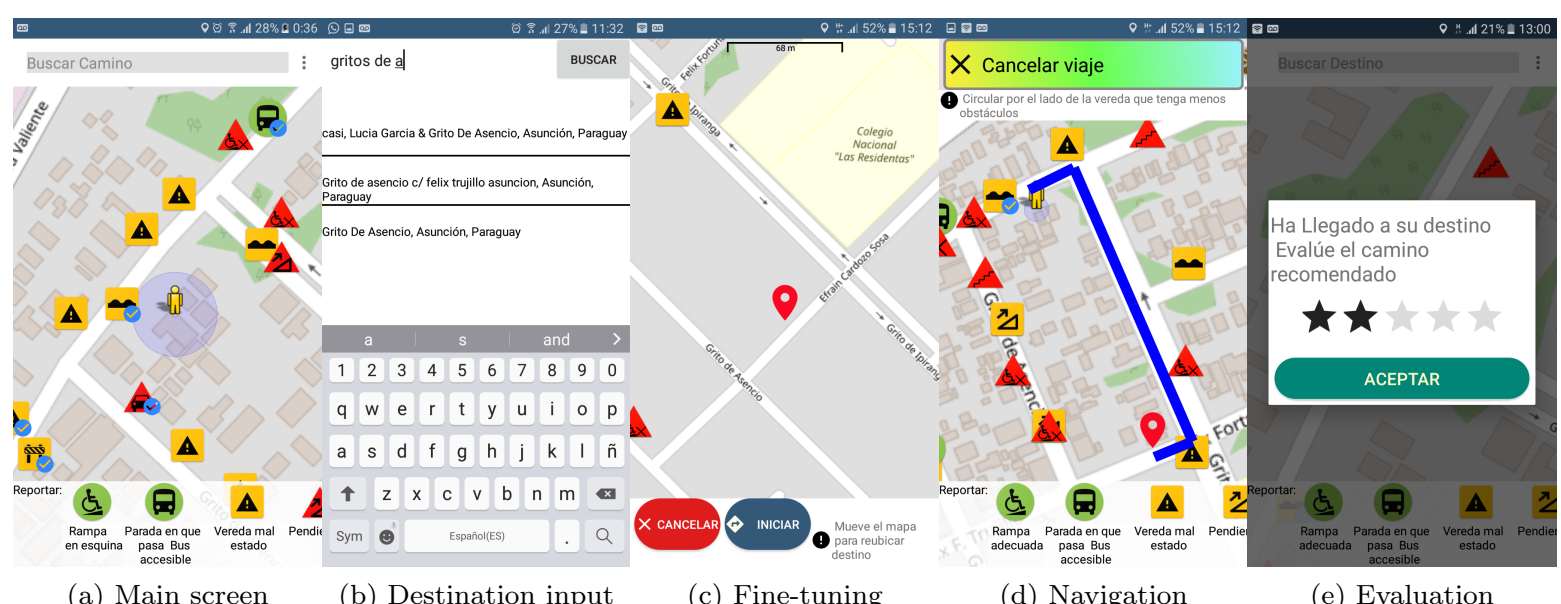

(a) Main screen

(b) Destination input

(c) Fine-tuning

(d) Navigation

(e) Evaluation

Figure 2: Route planning, navigation and evaluation

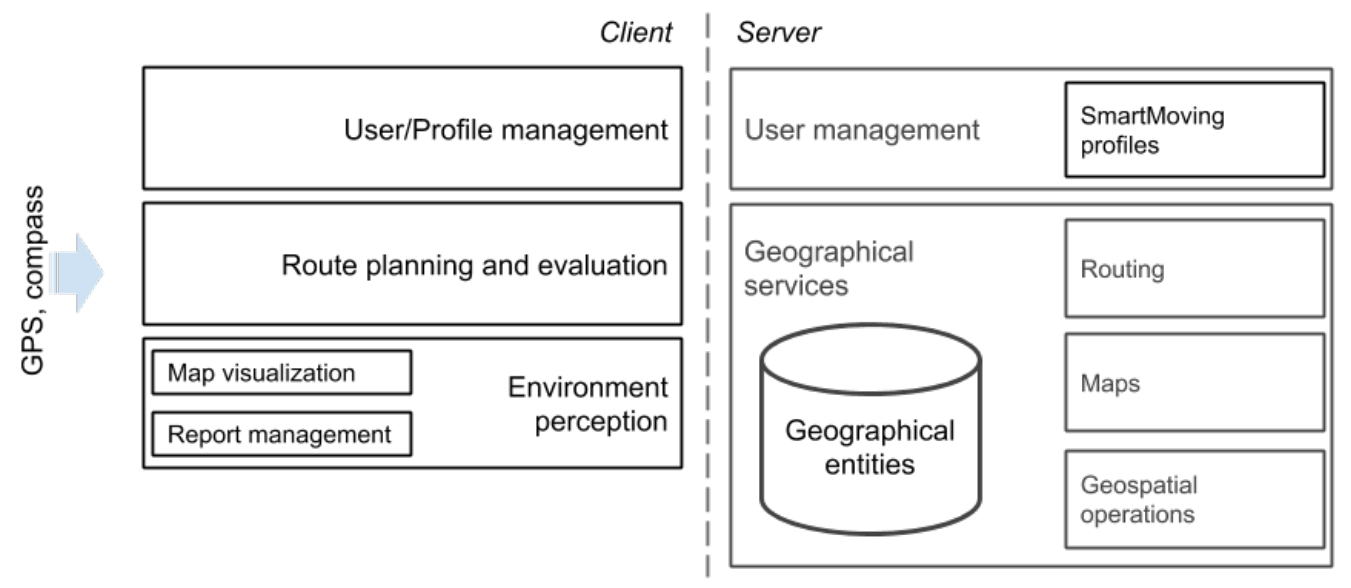

Figure 3: SmartMoving: Client and Server modules

\subsection{Architecture}

In this section we describe the architecture of SmartMoving, presenting module design and implementation details on both the server and the client. Figure 3 gives an overview of the client and server applications in terms of the modules that each one comprises.

\subsubsection{Client}

The client application resides on the smartphone device and is in charge of exchanging information with the user and sampling the GPS and compass sensors. Using the client application, a user can create their account, establish their identity and configure a mobility profile and the severity of each type of POI report. Once the profile is complete, the user can set a destination, get an automatic route recommendation according to their profile and navigate to reach the desired destination. Along the way, the user can create, confirm or resolve POI reports to keep an updated representation of the surroundings for other users.

The client comprises several modules that implement required functionalities and are described as follows.

User/Profile management. This module implements user interfaces to enable account creation, user identification and profile setup including characteristics of the user regarding mobility and severity of each POI report type.

Route planning and evaluation. This module implements destination selection, automatic route recommendation interface and user feedback. Automatic routes are recommended to minimize the expected difficulty for the user. This is realized using information from the profile of the user and relying this information to the server alongside the current position of the user at the origin and the desired destination.

Environment perception. This module is in charge of implementing the visualization that represents the static and dynamic aspects of the surroundings and the interactions needed so users can manage POI reports. 
Map visualization. This sub-module implements the flow of information from the application towards the user: a map visualization that models the surroundings: streets, sidewalks and POI reports contributed by users, the recommended route and the current position of the user.

Report management. This sub-module implements the flow of information from the user towards the application. It is in charge of the interfaces that allow users to create POI reports and confirm or resolve them.

Several types of POI reports are supported and listed as follows:

- Obstruction: this signals the presence of some kind of obstacle that makes traversing difficult or outright impossible(e.g., the presence of garbage, vehicles, scaffolding and others).

- Irregular pavement: any kind of pavement configuration that endangers wheelchair or crutch users.

- Steep ramp: excessively steep or excessively long ramp without resting landings that endangers wheelchair or crutch users.

- Stairs or steps: presence of stairs or steps without accessible alternative (e.g., accessible ramp).

- Inadequate street crossing: a lack of accessible ramps at one or both sides of a street crossing.

- Stop for accessible bus: bus stop served by accessible bus line.

\subsubsection{Server}

The server application resides in a central location and is online and reachable by all clients. It is in charge of keeping an up-to-date representation of the environment by accepting contributions from all users via the client applications installed in their devices, relaying this up-to-date representation to each client application on demand, subject to proper identification by the client. Additionally, it is in charge of recommending routes according to the updated representation of the environment and the parameters provided by the client to model origin, destination and severity of different POI report types.

The server comprises several modules that implement required functionalities and are described as follows.

User management. This module stores generic user account information and SmartMoving-specific profile information to identify users and model their mobility profiles.

Geographical services. This module implements geographical entity modeling and the necessary operations to manipulate these models and serve them as needed. Geographical entities are real objects of the environment such as streets, sidewalks, parks, bus stops, street lights and crossings. It is powered by a database of geographical entities.

Routing. This module implements automatic route recommendation between an origin and a destination. It takes into account the profile of the user and the characteristics of the environment to select a route that minimizes the expected difficulty of traversing the route. Relevant environment characteristics comprise static aspects determined by the geometry of nearby streets, sidewalks and related amenities and dynamic aspects determined by POI reports created by users.

Maps. This module implements a querying mechanism to retrieve geographical entities and pre-rendered map tiles to enable map rendering on the client.

Geospatial operations. This module implements low-level geospatial operations such as euclidean distance between entities, closest entity of a given type, entities within a polygon, which are needed by the Routing and Maps module.

\subsection{Implementation strategies}

This section provides some comments regarding the incidents report reliability as well as regarding the algorithm that SmartMoving uses for its automatic route planner.

In order to take care of the reliability of the data presented in SmartMoving, we have defined an incident scoring system, in order to verify the data that we obtain with the help of the users as well as to minimize the presentation of false data through the application.

SmartMoving users contribute by reporting the existence or persistence of incidents or obstacles, as well as by reporting the nonexistence or absence of them.

In SmartMoving, each POI report has one of the following three possible states: unknown, confirmed, and resolved. When a user creates a new POI report, it is assigned the unknown state. As long as the number of contributions for the existence or persistence of a POI report is less than 3, the unknown state is maintained. When 3 or more users have reported the existence or persistence of a POI report, it goes to the confirmed state. If a confirmed report receives an absence or nonexistence contribution, it goes to unknown state and 


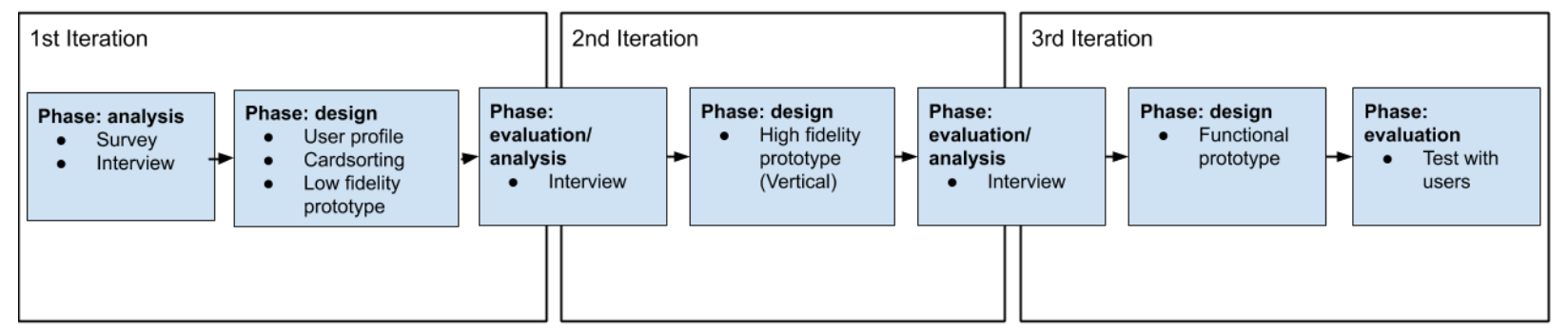

Figure 4: UCD process for the development of the SmartMoving interaction layer

remains in that state as long as it has less than 3 absence or nonexistence contributions. When obtaining 3 or more contributions of absence or nonexistence, the report goes to the resolved state.

On the SmartMoving map, POI reports with unknown and confirmed states are displayed. Confirmed ones are displayed with a blue check (see Figure 1 a and Figure $2 a$ ).

Nevertheless, we have not tested yet how the application behaves if multiple users are reporting the same or contradictory information at once.

For route planning, SmartMoving uses pgRouting 6 , which is a service that provides geospatial routing functionality. In particular, we use the K-Shortest Path algorithm, which provides the K shortest alternative paths from an origin to a destination. The value of $\mathrm{K}$ we use is 3, so SmartMoving starts calculating 3 shortest alternative paths from an origin to a destination. pgRouting defines a cost for each path, and with these costs it can calculate the shortest paths. In order to account for incident reports, SmartMoving adds an additional cost to each POI report in each path. The additional cost of each POI report depends on its configuration as minor or serious. Minor negative reports have an additional cost of 0.0001 and serious negative reports have an additional cost of 0.002 . Once the new costs are obtained, the 3 shortest alternative routes are ordered according to the new cost. The application will display the route with the lowest cost.

Therefore, SmartMoving always starts calculating 3 alternative shortest paths from an origin to a destination. Then, each of these 3 path's cost is recalculated taking into account its negative POI reports. Negative POI reports adds to a path's cost, therefore, at the end, the path with the lowest cost is selected. Taking into account that serious negative reports adds more cost than minor ones (0.002 vs 0.0001$)$ a path with several minor reports can be selected instead of a path with only one severe report.

It is worth mentioning that the goal of this work is not to create a new routing algorithm, nor to improve an existing one. We have simply reused an existing algorithm and used it in the context of an application that seeks to facilitate the pedestrian movement of people with reduced mobility. As we focus on pedestrian mobility, we have considered it important to use an algorithm that looks for shorter paths, and we have added penalty costs for paths with mild and severe negative reports.

It would be interesting to carry out tests with users to verify if, from their point of view, the SmartMoving path recommendation is good or not. Test results like these could help us fine-tune the penalty costs we initially assigned to minor and severe negative reports.

\section{Design of the SmartMoving interaction layer}

This section presents the planning and the User Centered Design (UCD) process for the development of the SmartMoving interaction layer.

\subsection{Process planning}

UCD is an iterative design process focused on the needs of the users. In addition to the stages of a classic process (analysis; design/implementation; and evaluation), UCD proposes a series of methods or techniques that can be used in each of the stages to obtain information from users about a product, its characteristics, contexts of use, etc. [4.

In this work, the process illustrated in Figure 4 has been followed. It consists of three iterations of classic processes, each with analysis, design and evaluation. In addition, the figure indicates the methods or techniques that have been adopted at each stage.

\footnotetext{
6 https://pgrouting.org/
} 
We considered two options for the first iteration of the process: performing usability inspections working with experts, which probably would have reduced the number of iterations of the process, or performing evaluations with users of the specific profile of the application (see Section 4.2), with which we consider it prudent to increase the number of iterations of the process somewhat. We managed to schedule the interviews with users in a shorter period of time and therefore we moved forward on that path. Therefore, the decision to perform three iterations in the UCD process was influenced by this decision to work with users rather than experts, as well as the availability of time to perform this work in the context of a research project.

The process carried out is summarized below, presenting the iterations and stages goals, and the justification for the choices of methods or techniques used.

In the analysis stage of the first iteration, we carried out a survey in order to obtain information on citizen perception about urban mobility problems in the city of Asunción, as well as on the state of the sidewalks. In addition, we carried out interviews in order to verify that there is an interest in having a tool like SmartMoving. In the design stage we have defined the SmartMoving user profile, in order to characterize them and be able to focus the application on that choice. Also, we have used the card sorting technique to categorize different types of obstacles that could be reported in the application. We have also developed a low-fidelity prototype of the user interface. In the evaluation stage, the low-fidelity prototype was evaluated by users who have the profile defined in the design stage, conducting individual interviews and applying the thinking aloud method.

The second iteration began with the analysis of the interviews from the previous validation stage, with the aim of identifying changes and improvements in the evolving user interface. Then, in the design stage we have used the high-fidelity prototyping technique, which implies the implementation of some of the SmartMoving functionalities, taking into account the information and recommendations obtained in the previous interviews. Later, in the evaluation stage, we have carried out a second round of individual interviews using the same thinking aloud technique, in which users were already able to interact with a functional prototype and expressed their opinions regarding it.

Similarly, the third iteration began by analyzing the interviews carried out in the previous validation stage to identify areas for improvement. Next, a first complete version of SmartMoving was developed (see Figure 1 and Figure 2, with all its functionalities implemented and taking into account the feedback received from the users interviewed. Finally, for the validation stage, a test with users was carried out.

The three iterations of the DCU process are presented below, including information on the data collected, results, and analyzes performed.

\subsection{First iteration}

The analysis stage began with a survey and interviews with the aim of exploring people's perception about the problem of pedestrian mobility in Asunción. The survey had a broader goal related to obtaining information on the public perception about urban mobility problems in general, and also included questions related to pedestrian mobility. 589 people answered the survey and $72 \%$ of them consider that the condition of the sidewalks is a serious or very serious problem [11.

The poor condition of the sidewalks can especially affect people with reduced mobility. For this reason, we proceeded to conduct an interview with a representative of the organization ParIgual 7 , whose goal is to claim the rights that people with disabilities should have, one of them being accessibility. This interview allowed us to obtain a better understanding about the problem as well as suggestions about the application to be developed from a representative of potential users. In the interview, the existence of an interest in having a tool such as SmartMoving was verified, as well as ParIgual's interest in collaborating in the project, participating in the UCD process, and in establishing contact with other people or institutions that could collaborate in the process.

In the design stage we have defined an initial user profile, with the aim of refining it later in the interviews of the validation stages of the first and second iteration of the process. The initial user profile for SmartMoving is that of people with reduced mobility (who use a wheelchair, cane, walker, etc.) and who own a smartphone.

On the other hand, also as a design activity, we have developed a low-fidelity prototype of SmartMoving using the NinjaMock tool 8 , a web application to build mockups. The design of this prototype was built taking into account the comments and recommendations of the interview that was carried out in the analysis stage. This prototype is made up of 20 mockups 9

Figure 5 shows the SmartMoving main screen, in its low-fidelity prototype version. In it, the map of the surroundings of the user's location and the POI reports that were previously reported by other users are displayed. The POI reports represent incidents on the sidewalks and following the analogy of the traffic

7 https://www.facebook.com/parigual

8 https://ninjamock.com/

9 https://drive.google.com/open?id=1QZ5MeThGV9-i04Hp_SSmj02MSSbjqM41 


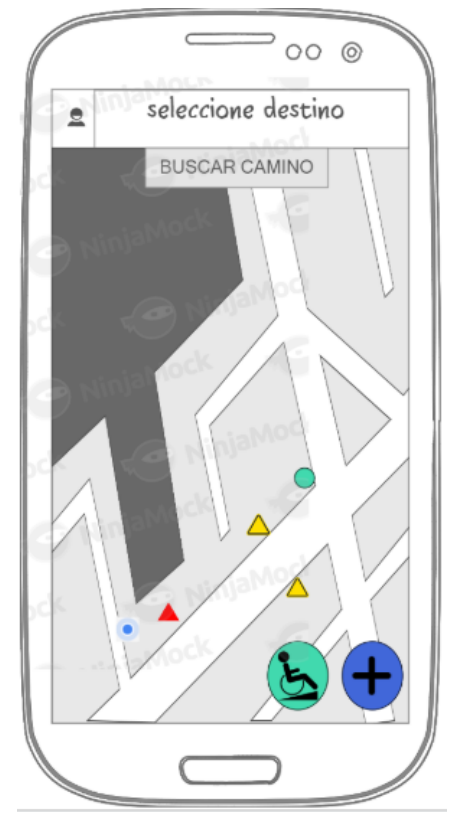

Figure 5: Main screen of the SmartMoving low-fidelity prototype

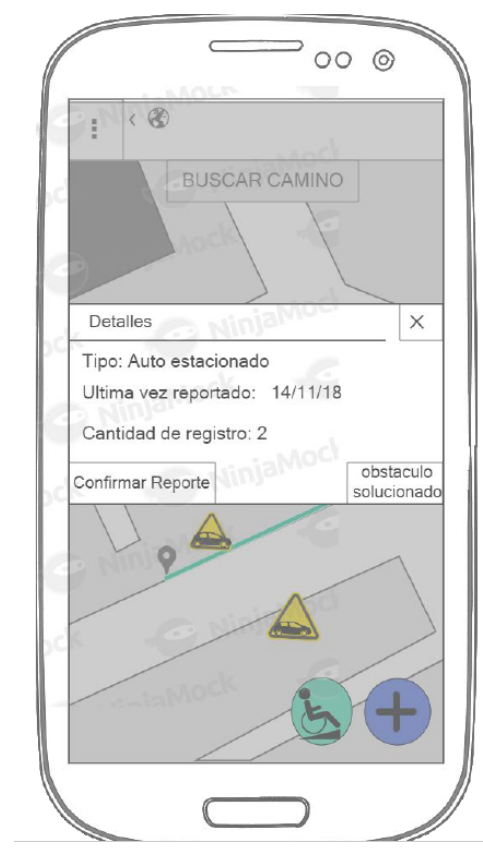

Figure 6: Screen with details of a POI report

light they are represented on the map with markers of different colors and shapes: the circular green marker represents a positive report, the triangular yellow marker represents a mild negative report and the triangular red marker represents a severe negative report.

If the user presses any of the POI reports that are displayed in Figure 5, he will go to see the screen of Figure 6, in which more details about the selected POI report are presented. In addition, the user can contribute to the SmartMoving information confirming the existence and persistence of the POI report or indicating that this POI report is already resolved and is no longer an obstacle. For these purposes, Figure 6 presents two buttons, one to confirm the existence of the incident/event and the other to mark it as resolved.

With the low-fidelity prototype ready, we proceeded to the evaluation stage, conducting interviews with users of the defined profile. Three men participated, two of them mobilize with a wheelchair and one with a cane. The audios of the interviews and photographs can be consulted at the following URL 10

During the evaluation, we showed the users the slides of the low-fidelity prototype (see ${ }^{11}$ ) and proposed them to explain how they would solve certain tasks, without the help of the evaluators. In other words, users were asked to use the thinking aloud technique to explain how they would proceed to complete a task that the evaluator proposed and also to express doubts about what they did not understand. This was to help us understand if the design was understandable. The tasks were as follows:

- Task 1. Showing the interviewees Figure 5, they are asked to identify the POI reports that are on the map.

- Task 2. Showing the interviewees Figure 5, they are asked to explain what they interpret from the green, yellow and red markers.

- Task 3. By showing users the sequence that appears in Figure 7, they are asked to explain how they would proceed to create a new POI report.

- Task 4. Showing the users Figure 6, they are asked to explain how they would proceed to confirm the persistence of a POI report, or to mark it as resolved.

- Task 5. By showing users the sequence that appears in Figure 8, they are asked to explain how they would proceed to find a path to a certain destination.

Table 1 presents the results. Cells containing Yes indicate the user was able to perform the corresponding task while cells containing No indicate the opposite.

As can be seen, with respect to Tasks 1 and 2, it was easy for the interviewees to recognize the reports that were on the map, as well as to interpret what the green, yellow and red markers represent.

\footnotetext{
10 https://drive.google.com/drive/folders/1MvQe9ifDVLVijBFq50xsfxcoLLIGZjpe?usp=sharing

11 https://drive.google.com/open?id=1QZ5MeThGV9-i04Hp_SSmj02MSSbjqM41
} 


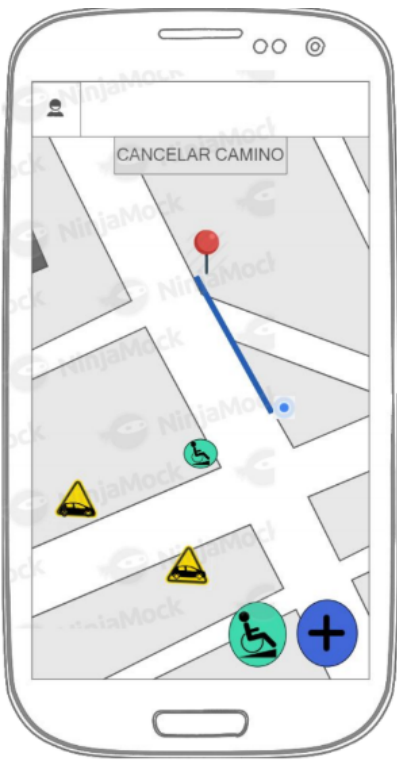

(a) Main screen

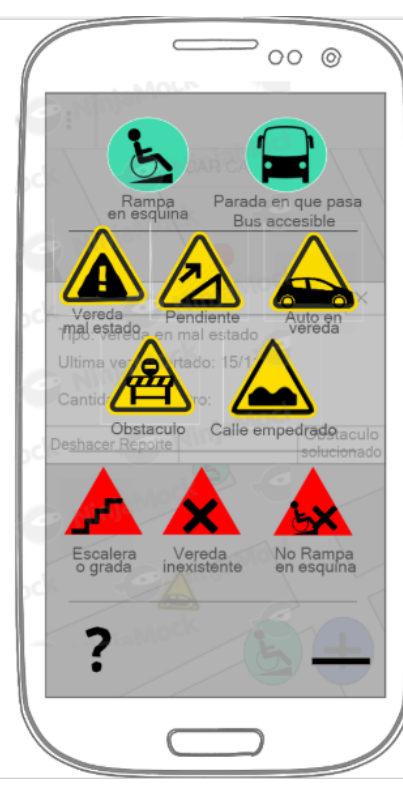

(b) Incident to report

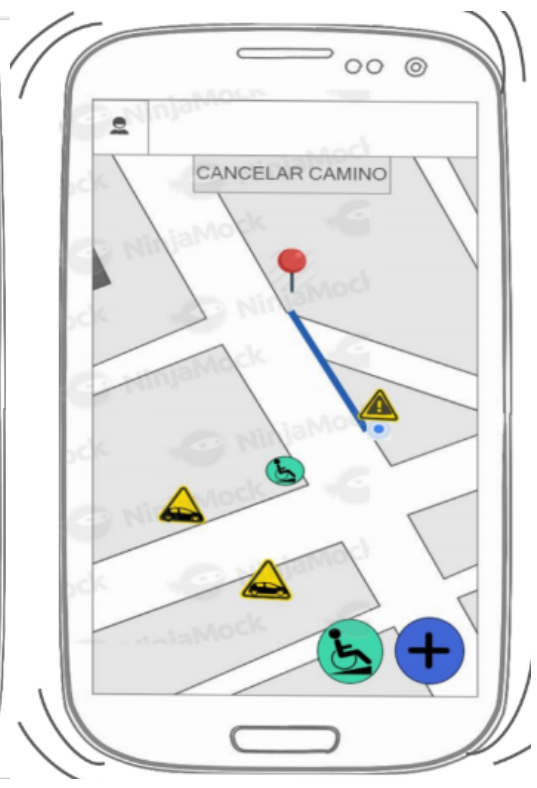

(c) New incident in map

Figure 7: Sequence of screens to perform Task 3

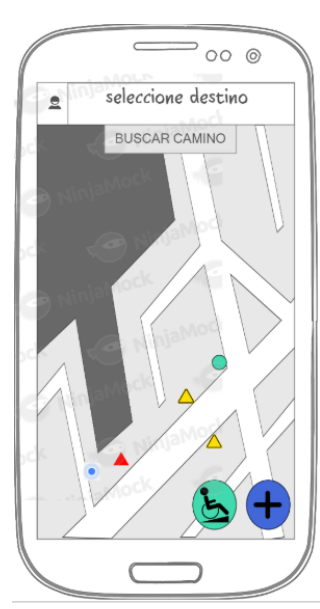

(a) Main screen

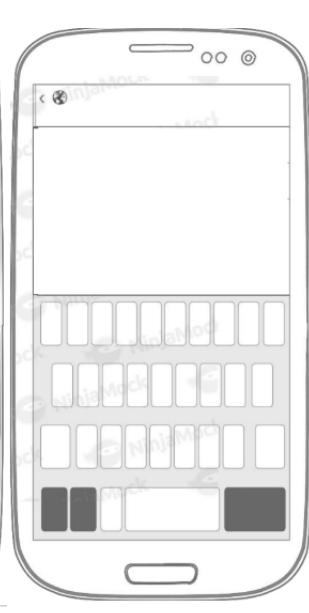

(b) Destination input

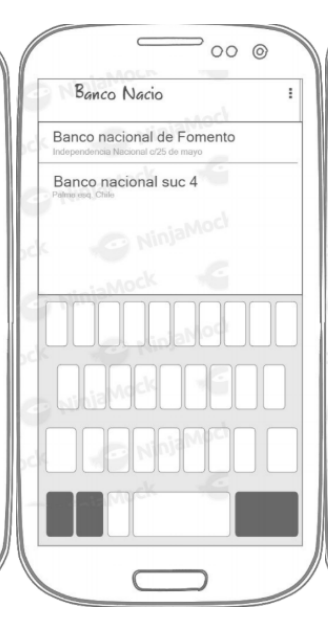

(c) Destination selection

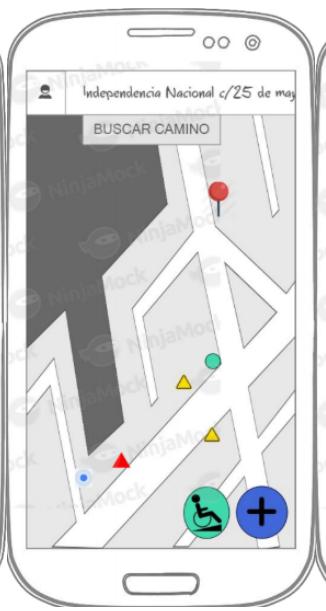

(d) Destination tuning

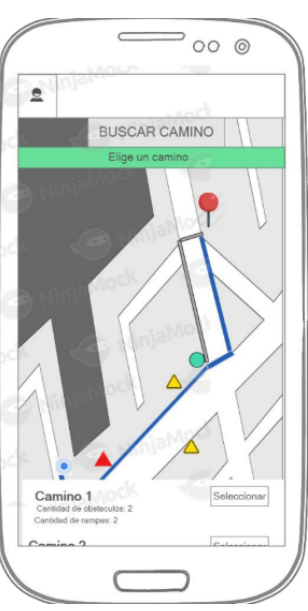

(e) Navigation

Figure 8: Sequence of screens to perform Task 5

Tabla 1: Results of the low-fidelity prototype evaluation tasks

\begin{tabular}{lccc}
\hline & Interviewee 1 & Interviewee 2 & Interviewee 3 \\
\hline Task 1 & Yes & Yes & Yes \\
\hline Task 2 & Yes & Yes & Yes \\
\hline Task 3 & Yes & No & No \\
\hline Task 4 & Yes & No & No \\
\hline Task 5 & No & No & No \\
\hline
\end{tabular}



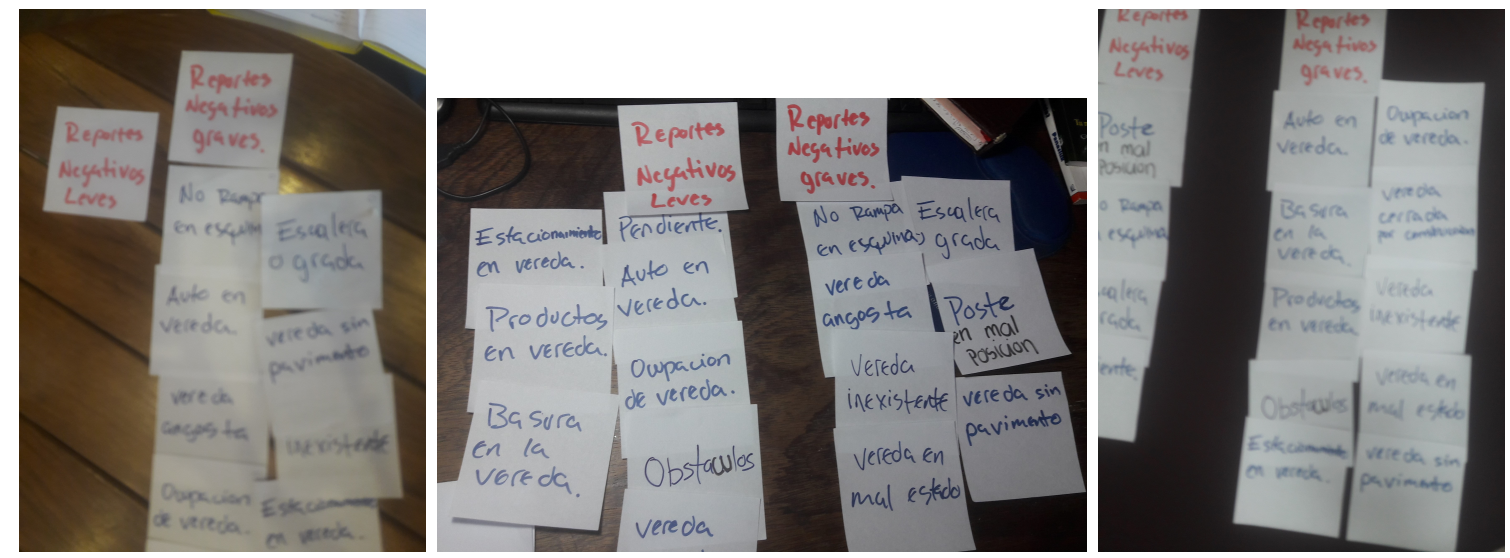

Figure 9: Card sorting of mild or severe negative POI reports

Regarding Task 3 (create a POI report), only one of the interviewees could indicate how to do it. The other two needed the help of the evaluators. The two buttons that generated confusion are shown in Figure $7 \mathrm{~A}$. Instead of selecting the blue button with the plus sign to get to the next screen that allows selecting the type of report to be created, they selected the green button with the ramp symbol.

Again, in Task 4 (confirm or resolve a POI report) two of the interviewees were unable to complete the task without help from the evaluators. The two buttons found in Figure 6 were not clear for the interviewees to determine which they should select, either to confirm a POI report or to mark a POI report as solved. The problem that we deduced from the behavior of the two interviewees is that there was no help to indicate to the user how they can contribute with this type of POI report.

Finally, the three interviewees found it difficult to carry out Task 5 (find a path). The button that generated confusion appears on Figure 8 $\mathrm{a}$. The three interviewees decided to start the task pressing the "Find a Path" button. Instead, as a first step, they had to press the "Select path" input control. One of the interviewees told us that the button was distracting because of where it was located.

On the other hand, during the interviews, the card sorting technique was used to categorize negative reports according to whether they were mild or severe. The card sorting consists of the presentation of a series of cards that contain concepts that users must organize according to their criteria. The goal of categorizing concepts is to facilitate the understanding of the user. In our case, we presented the different types of negative reports (non-existent ramp, sidewalk in poor condition, non-existent sidewalk, vehicle on the sidewalk, stairway, slope, etc.) and users had to categorize them according to whether they were minor or serious (see Figure 91. In the category of mild negative reports, there were no coincidences among the interviewees. On the other hand, in the category of serious negative reports there were three coincidences in the following types of reports: sidewalk in poor condition, non-existent sidewalk and sidewalk without pavement.

We conclude that the SmartMoving low-fidelity prototype interface needs some improvements in terms of user understanding. A design review and proposing solutions that solve each problem are essential. In this sense, in the analysis stage of the second iteration, the problems encountered are summarized and solutions are defined for each one of them. Suggestions and comments from users are also analyzed. It is also defined how to proceed with categorizations of negative reports.

\subsection{Second iteration}

The second iteration began, again, with an analysis stage based on the interviews in which the SmartMoving low-fidelity prototype was evaluated and the data obtained from the card sorting exercise during the validation stage of the first iteration. We analyze the problems found, as well as the suggestions and comments of the interviewees to identify coincidences and make decisions about the changes to be incorporated into the prototype.

The analysis carried out to identify problems consisted of transcribing the notes that the evaluator took during the evaluation of the low-fidelity prototype, together with what was expressed by the interviewees with the thinking aloud technique, in order to find coincidences and identify problems. In order to illustrate the analysis process, we present Table 2, as an example. This table presents the interviewer's notes and the expressions (recorded) of the interviewees in relation to slide 6 of the low-fidelity prototype of SmartMoving (see Figure 5). From these notes and recordings a problem with the prototype is identified. Tables containing all notes, recordings, and problem identifications for the initial low-fidelity prototype of SmartMoving can 
Tabla 2: Example of problems identification from interviewer notes and interview recordings, for slide 6 of the SmartMoving low-fidelity prototype (see Figure 5 ).

\begin{tabular}{|c|c|c|c|c|}
\hline $\begin{array}{l}\text { Slide or } \\
\text { screen \# }\end{array}$ & Interview 1 & Interview 2 & Interview 3 & Problem \# \\
\hline $\begin{array}{l}\text { Slide } 6 \\
\text { of the } \\
\text { low-fidelity } \\
\text { prototype } \\
\text { (see } \\
\text { Figure 5) }\end{array}$ & $\begin{array}{l}\text { Interviewer notes: } \\
\text { Does not recognize } \\
\text { what the green } \\
\text { icon on the screen } \\
\text { indicates and only } \\
\text { recognizes that the } \\
\text { colors indicate alert. } \\
\text { Recording: "This } \\
\text { wheelchair would } \\
\text { represent the options } \\
\text { of road, perhaps, } \\
\text { or help"; I guess } \\
\text { the colors are like a } \\
\text { traffic light". }\end{array}$ & $\begin{array}{l}\text { Interviewer notes: } \\
\text { He confuses the } \\
\text { button to report } \\
\text { corner ramp and only } \\
\text { recognizes some alert } \\
\text { in relation to the } \\
\text { colors of the reports. } \\
\text { Recording: "The } \\
\text { red triangles would } \\
\text { indicate obstacles, } \\
\text { paths that are not in } \\
\text { good condition, the } \\
\text { green ones I think } \\
\text { show me ramps"; } \\
\text { "The yellow triangles } \\
\text { could indicate that } \\
\text { the sidewalks are in } \\
\text { poor condition". }\end{array}$ & $\begin{array}{l}\text { Interviewer notes: } \\
\text { He recognizes the } \\
\text { colors of the POI } \\
\text { reports, but does } \\
\text { not know what they } \\
\text { refer to. Recording: } \\
\text { "They show me some } \\
\text { streets. What I don't } \\
\text { see is the name of the } \\
\text { streets "; "What is } \\
\text { in yellow are signs, } \\
\text { what is in blue or } \\
\text { green are ramps"; } \\
\text { İ don't understand } \\
\text { the dark part". }\end{array}$ & $\begin{array}{l}\text { Problem } 3: \\
\text { Interviewees do not } \\
\text { identify what type } \\
\text { of incident/event the } \\
\text { colored markers on } \\
\text { the map refer to. }\end{array}$ \\
\hline
\end{tabular}

be found at the following URL 12 .

Below we present all the problems encountered by the interviewees in the evaluation of the low-fidelity prototype, with their respective solutions.

- Problem 1: people with reduced mobility may find it difficult to move around and use the mobile device at the same time. If many steps are required to use the SmartMoving capabilities, this can be a problem for people. Solution: minimize the required steps to create reports and to use the pedestrian path recommendation.

- Problem 2: when performing Tasks 3 and 5, some buttons found on the main SmartMoving screen (see Figure 5) were confusing. The buttons are the green accessible ramp icon, the blue icon with the plus sign (which was intended to allow the other types of reports to be reported), and the "Find path" button (which requires that users enter the destination before pressing it). Solution: in Figure 5 . replace the green button with the ramp and the blue with the plus icon by a window, located at the bottom of the screen, containing all types of POI reports and scrolling from left to right to view them all, thus minimizing the steps to create a report. Replace the "Find path" button with one that is visible to the user only after the user has selected the destination.

- Problem 3: the colors of the markers that appear on the maps to indicate alerts, events or obstacles previously reported, refer to their severity (see Figure 5) according to logic of the traffic light. However, the interviewees do not know what type of incident/event they are referring to. Solution: include the icons representing the POI reports on the map, as shown in Figure $7 \mathrm{~b}$.

- Problem 4: Difficulty in interpreting the drop-down window found in Figure 8p. The window contains the paths that the application recommends and the user should press the "Select" button to choose one of the recommended paths. One of the users stated that this window seemed unnecessary to him. Solution: remove the drop-down window and directly show the best recommended path on the map.

- Problem 5: on the screen that shows details of a selected report (see Figure 6) some confusion was generated regarding the buttons to confirm if a reported event/incident is still valid or indicate that the problem has already been solved. Solution: change the display of the buttons in that window.

On the other hand, the following aspects were clear to most of the interviewees:

- The colors that indicate levels of difficulty: red, yellow and green.

\footnotetext{
12 https://docs . google.com/spreadsheets/d/1cVuT131ITEnhhkJ4xtDpEQyAWUrPuYt0CZ1AajM36Ds/edit?usp=sharing
} 
- What the report icons represent: ramp, stairs, parked car, etc.

- The suggested path, marked in blue, the point of origin and the destination (see Figure 8p).

- The icon that indicates where the user or the person who is using the application is.

- Suggestions of paths made by the application.

It is also relevant to analyze suggestions and comments of users. User suggestions were the following:

- Suggestion 1: Do not use the same geometric shape, i.e. the triangle, to represent minor and severe negative reports.

- Suggestion 2: Incorporate a motivating detail, message, or positive reinforcement to encourage users to create, confirm, and / or resolve reports.

- Suggestion 3: Allow the user to relocate a created report.

- Suggestion 4: Be able to create reports using voice commands.

- Suggestion 5: Be able to create reports in locations other than the user's current location.

- Suggestion 6: Deploy a web version of the application.

We decided to implement suggestions 1 and 3. For suggestion 1, we decided to use yellow square markers for mild negative reports and keep the red triangles for severe negative reports. We recognize the importance of all other suggestions, but because of the scope and deadlines of this project, we decided to incorporate them in future versions of SmartMoving.

Regarding comments, users stated that the purpose of the application was clear to them, that they found the application useful, and that, in general, they intuitively understood how to use the tool. They also expressed the need and importance of having this type of digital services for two main reasons: first because of the novelty that this means in the field, since there are already many tools of this type, but all of them are aimed at people who move in cars, bicycles or public transport, and second because of the poor state of the sidewalks and the spaces for people who walk. They emphasized the numerous obstacles and difficulties they have to walk in Asunción, even when the distance is short.

On the other hand, in relation to the results of the card sorting exercise, as there was almost no coincidence in the categorization of mild and severe negative reports, we conclude that it is likely that said categorization is dependent on the type of mobility of each user, or each user in particular. Therefore, it was decided to add a functionality so that each user can categorize negative reports, according to their opinion, as minor or serious.

In the design stage, the high-fidelity prototype was developed, implementing improvements based on user suggestions and solutions to the problems found in the analysis stage. The SmartMoving prototype was developed for Android devices and in this iteration the functionalities of visualizing the map of the surroundings of the user's location were implemented, including the visualization of POI reports (markers). The POI report creation functionality was also implemented.

Figure 10 shows some screenshots of the high-fidelity prototype. The first screen (Figure 10a) shows the map of the surroundings of the user's location, including markers. Below is the scrolling window that includes all types of reports and allows the creation of any of them. The second screen (Figure 10p) displays a message with instructions to locate a report. The third screen (Figure 10 c) shows a response message when the user created a POI report.

In the evaluation stage, three individual interviews were carried out again. One of the interviewees had already participated in the first iteration. There were two male participants and one female, all three of them mobilized with a wheelchair; one of them uses a motorized chair. The audios of the interviews and photographs can be consulted at the following URL13

New interviewees, who did not participate in the first iteration, were briefly introduced to the prototype and the functionalities that were implemented. On this occasion, the task consisted in asking users to explain what steps they have to follow to create a POI report, using the thinking aloud technique, and also to express doubts about what they do not understand. The starting point was Figure 10 a.

At the end of this iteration we conclude that the high-fidelity prototype is generally well understood by the interviewees. The number of problems found is less compared to the first iteration. However, some modifications are necessary to achieve a more intuitive understanding of certain steps to follow, taking into account the suggestions and behaviors that users manifested during the interview. The problems found are summarized in the analysis stage of the third iteration, and solutions are defined for each of them.

\footnotetext{
13 https://drive.google.com/drive/folders/1KE41aQfxPXkm1xgQDB5V25EUyy794E8j?usp=sharing
} 


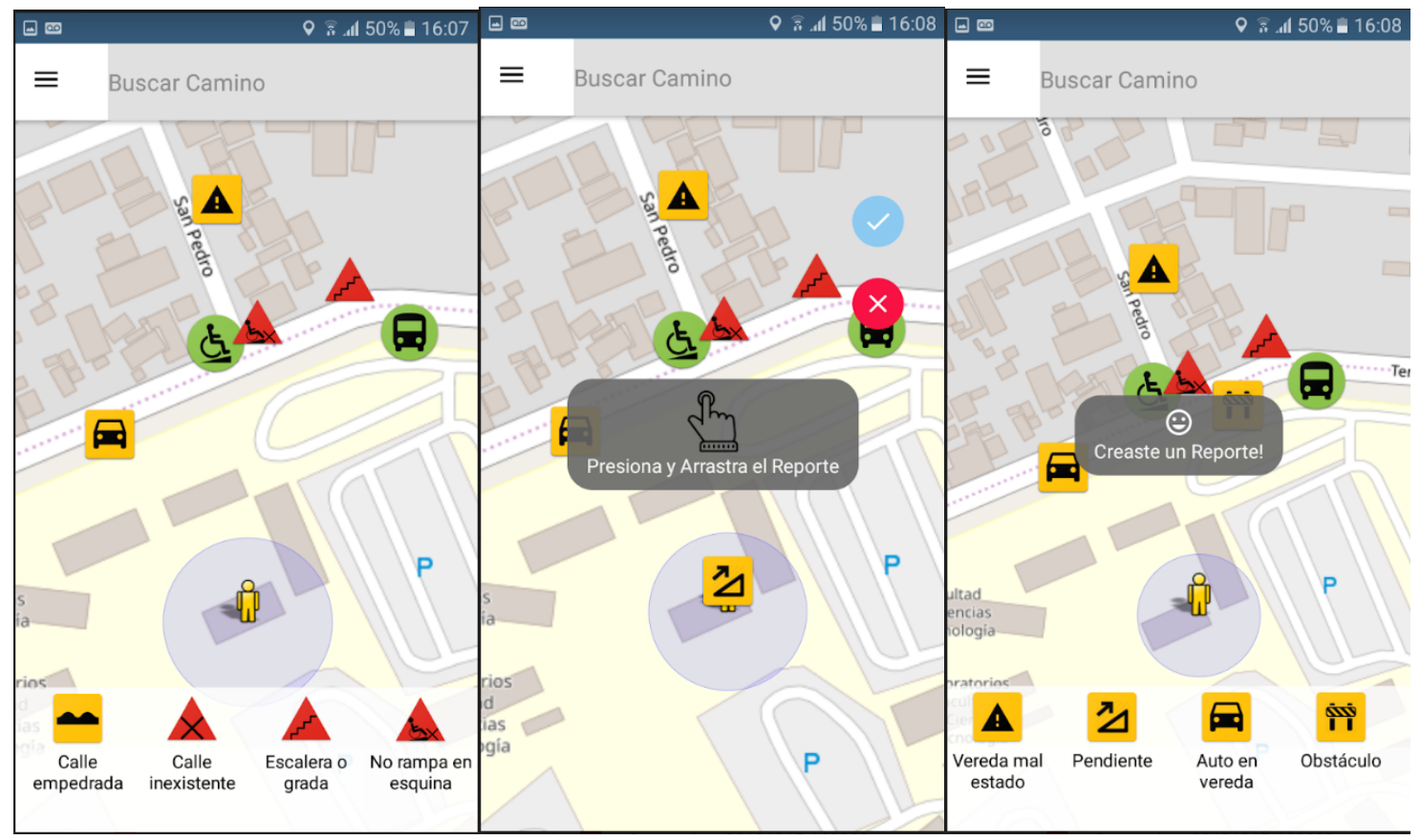

(a) Main screen

(b) Incident location

(c) Incident reported

Figure 10: SmartMoving high-fidelity prototype

\subsection{Third iteration}

In the third iteration, the analysis stage corresponds to the analysis performed over the data collected during the interviews in which the high-fidelity prototype was subjected to evaluation. The tables containing the notes, recordings and problem identifications of the SmartMoving high-fidelity prototype, can be found at the following URL 14 . In summary, below we present the list of the problems found by the interviewees, their suggestions and their respective solutions.

- Problem 1: the interviewees did not pay attention to the messages that indicated how to create and locate a POI report (see Figure 10b). Solution: improve the display of messages.

- Problem 2: only one of the interviewees was able to perform the exercise of pressing and dragging a marker to relocate it on the map. Suggestion: relocate a marker by clicking on the map, instead of dragging it. Solution: we decided to take the suggestion as it reflected the behavior of two of the interviewees.

- Problem 3: Two of the interviewees found the report of corner ramp confusing as there may be corner ramps that are not adequate, as well as there may be accessible ramps that are not located on corners. Solution: change the name of the POI report type that caused confusion, replacing "Corner ramp" with "Proper ramp", as well as the name of the report "No corner ramp" for "Inadequate or nonexistent ramp".

- Problem 4: the three interviewees expressed their interest in connecting with other social networks to make login, since it is difficult for them to remember the registration data, which is required to enter the application. However, the application needs specific data about their type of mobility. Solution: Once the first login has been registered and performed, the credentials will be saved and they will be automatically filled in the input fields when they want to start a session again.

Regarding suggestions, one of the interviewees expressed interest in being able to publish the POI reports that are created in SmartMoving on their social networks. We found this suggestion interesting, so we decided to comment on it to the other two interviewees. With everyone's consent, we decided to implement this functionality, which allows users to publish the POI reports they create in SmartMoving on Facebook and Twitter. Another suggestion whose implementation we decided to postpone until future versions of SmartMoving was that the application verifies the location of the reports, so that it is not possible to report

\footnotetext{
${ }^{14}$ https://docs.google.com/spreadsheets/d/1cVuTl31ITEnhhkJ4xtDpEQyAWUrPuYtOCZlAajM36Ds/edit?usp=sharing
} 


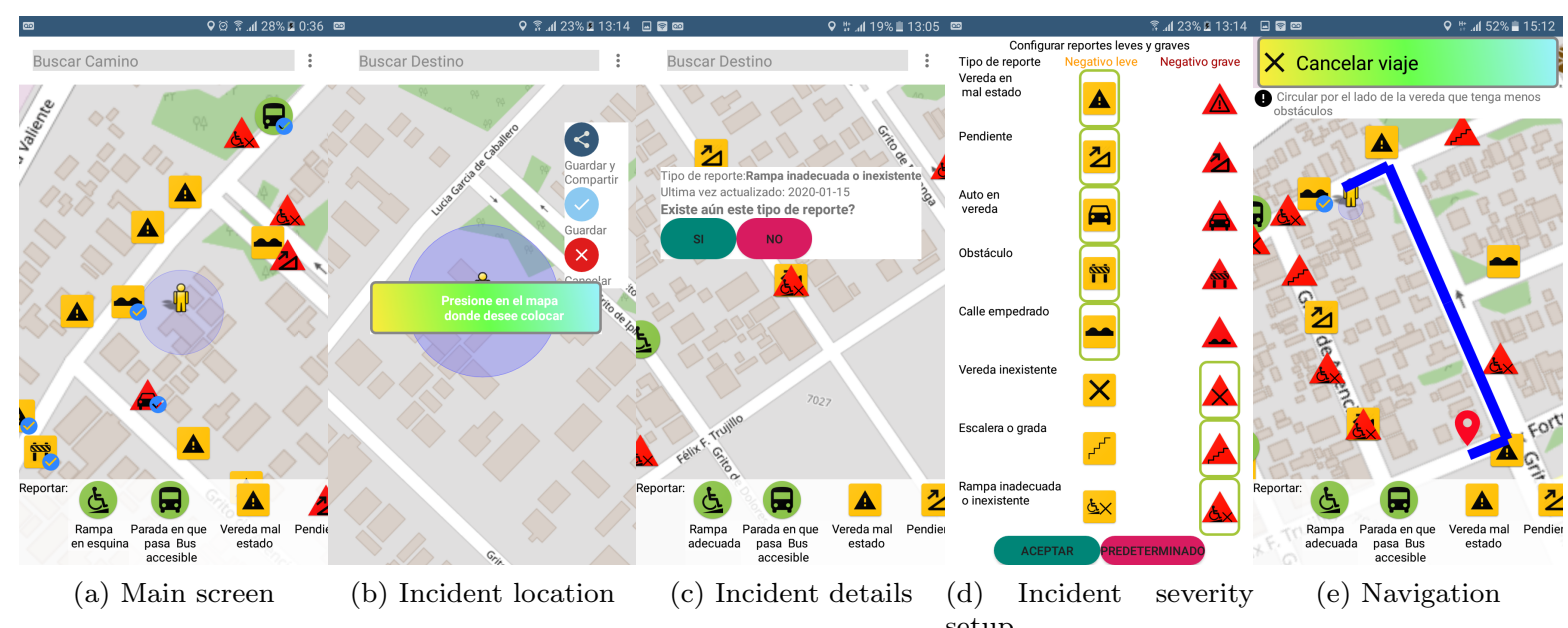

Figure 11: First functional version of SmartMoving

incidents or events in places where it does not correspond, such as reporting an accessible bus stop within a building.

Again in this round of interviews, the participants mentioned their difficulties when moving around the sidewalks, due to the different obstacles that are usually found on them. We emphasize that the three participants commented that using an application such as SmartMoving would be of great help to them to move daily, especially in areas they do not know since they could visualize in advance what are the obstructions they found on a road, and thus they could choose a route that make it easier for them to get to their destination.

The design stage of this last iteration consisted in the development of all the remaining functionalities of the high-fidelity prototype according to the decisions taken in the analysis stage.

Figure 11 shows some screenshots of the first functional version of SmartMoving. They are related to the functionalities of displaying on the map, creating reports and configuring negative reports (categorizing them as minor or serious).

After implementing the first functional version of SmartMoving, in the evaluation stage, the test-with-users technique was used. The test-with-users technique consists of carrying out validation tests with users, to check if there is an adequate design and to identify difficulties. The test was carried out in natural setting involving users [12, to determine how the product will be used in real life and obtain a qualitative assessment from users.

Tests were performed with three users, the first user had previously participated in the first iteration, the second participated in the second iteration and the third participated in the first and second iteration. Three male users participated, two use wheelchairs and one uses a skateboard.

The user test in this last iteration was performed using the functional version of SmartMoving. Each test was carried out independently of the others in terms of time and place, taking into account the availability and convenience of the participants. Each user used the SmartMoving application emulated on an Android smartphone (version 23).

Before performing the test, the evaluators uploaded information on the state of the sidewalks in the area of the city where each test was performed. Later, during the SmartMoving test, the evaluator explained to the user how the test will be carried out, remembering the functionalities of the application. First, the user categorized the mild and severe negative POI reports at their discretion. After categorizing the POI reports, the user made a request for a recommendation on the way.

The evaluator accompanied the users while they used the application. Applying the thinking aloud technique, each user gave their opinion. Positive comments were obtained about the application, expressing interest to start using it. Users also commented that the application will help them move through the streets and that they do not know of another application equal to SmartMoving.

Once the tour was finished, the evaluator gave each user a questionnaire about the usability of the application they used in the test. The CSUQ [13, 14] questionnaire was used. The questions can be found at the following URL 15 . We chose this questionnaire as it has a high degree of reliability and is considered adequate to evaluate this type of applications in mobile environments that require interaction from users.

\footnotetext{
15 https: //drive.google.com/open?id=1mD9FMTHmvr59U3kRCZfPRFqZGfKRcLba1TC-p9Fx-wU
} 
The CSUQ questionnaire contains a total of 17 questions. Questions 1 to 6 are aimed at measuring the usefulness of the system; then, questions 7 to 12 aim to measure the quality of the information; questions 13 to 15 are to evaluate the quality of the interfaces; and one last question, ask for comments/suggestions about the SmartMoving application. A Likert scale was used, which has 7 points, with 1 being the best score (totally agree) and 7 the worst score (totally disagree).

In the first group of questions, related to the utility of the system, an average of 1.55 was obtained, which is interpreted as agreeing with a positive perception of the utility of the system. In the group that corresponds to the quality of information, an average of 1.61 was obtained, which is also interpreted as agreeing with a positive perception of the quality of the information. Then, we have the group to measure the quality of interfaces, obtaining an average of 1.16, in this case corresponding to being totally in agreement with a positive perception of the quality of the interfaces. Finally, the general average of responses is 1.47 , which corresponds to agreeing with a positive perception of the general usability of the application. This usability assessment was previously presented in [6].

As can be seen, the results of the CSUQ questionnaire were positive in all dimensions. In particular, the result of being in complete agreement with the quality of the interfaces is reflected in the continuous participation of users throughout the participatory design process. These results support the goal of our solution, which is to obtain a prototype of the SmartMoving application that is intuitive, easy to use and that provides the appropriate information for the user profile that we previously defined.

\section{Findings and Discussion}

Taking into account the various improvements that were introduced from the initial SmartMoving prototype to the final functional prototype, we can say that the adoption of the UCD process was successful and useful, especially when addressing a specific user profile such as people with reduced mobility, an audience for which we had not previously implemented solutions.

When deciding to carry out a UCD process, an important decision is related to how many iterations to carry out until a functional product is achieved. In this sense, as previously mentioned, we have had the intention to speed up and reduce some iteration of the UCD process using usability inspection techniques with the help of experts in accessibility standards. However, with more iterations, the same result has been reached, since in the last stage of the process the understanding of all functionalities was achieved by users.

Regarding which specific techniques to use at each stage of the UCD process, we received recommendations from a psychologist familiarized with various techniques to collect data from users. Nevertheless, some decisions were adjusted as the work progressed, for example, initially we had planned to hold focus groups instead of individual interviews with users, but the great difficulty of coordinating several people with mobility problems to attend together at a specific place and time, due to the difficulty of getting around in Asunción, led us to adjust the initial plan and decide we will go to the place indicated by them, therefore replacing focus groups with individual interviews.

At different stages, several stakeholders participated in the UCD process for the development of SmartMoving. At the analysis stage of the first iteration of the UCD process, a citizen survey helped us confirm that sidewalks condition is considered a serious problem in Asunción, and an interview with a representative of an institution that works for the rights that people with disabilities should have (ParIgual) allowed us to confirm an initial interest for having an application like SmartMoving. At the design stage of the first iteration, a card sorting exercise with reduced-mobility users helped us decide how to implement the categorization of negative reports. At the evaluation stage of all three iterations, reduced-mobility users participated by means of interviews and user tests that helped us improve the SmartMoving prototype iteratively. Thanks to the active participation of users in the UCD process we were able to better understand the user and their needs, knowing the difficulties they have when moving through the streets of Asunción and the preferences that they would like to see in an application like SmartMoving.

Therefore, we believe that the resulting version of SmartMoving will be useful for the initially defined user profile. Of course, much more effort from different actors will be necessary to make Asunción an inclusive city, but SmartMoving can at least help people with reduced mobility to be better informed about the state of the sidewalks to facilitate their movement.

It should also be noted that all evaluations that were already performed were executed in the context of a UCD process for the development of the application. This was the case because our main goal was to develop an application useful and easy to use by people with reduced mobility. However, we still have to evaluate the application in real use, considering cases such as reporting from multiple and simultaneous users, reliability of the information retrieved from users, willingness of the citizenship in providing information, among other aspects to be considered.

One question that remains to be better explored is whether there would be differences between users who 
already know, even partially, a location, and others who do not know it at all. As previously mentioned, our interviewees remarked that the application would be especially useful when the user is in unknown or less frequented places.

By means of its use of crowd sourced data, necessary for an up-to-date representation of the environment, SmartMoving is very sensitive to the cold start problem. This is especially true for the general problem of city-wide automatic accessible routing. Mitigation strategies involve incentives to broaden the user base (e.g., by offering value not only to users with disabilities but to all kinds of users) or scenario modifications that reduce the scope of its applicability (e.g., in the context of closed communities). In fact, our interviewees have mentioned the importance of offering some positive incentive or reinforcement when users create or update reports.

Offering value to more kinds of users could be achieved by extending the application to support mixed modes of transportation (e.g., offering routes that incorporate bus ride segments) or new types of users (e.g., bicycle users which can also be impacted by lack, decay or misuse of bicycle-friendly infrastructure).

Contributions by SmartMoving users are evidence of citizen movement through areas of the city and they could provide a valuable source of information regarding the state of the city infrastructure and the way this infrastructure is used. POI report analysis could have as a result indicators that describe accessibility aspects for streets, neighborhoods or schools. POI reports could be used alone or as part of a set of sources to compute indicators related to infrastructure use, misuse and damage. Map visualization services that take these indicators as input would constitute a valuable tool for decision makers to understand where to focus infrastructure enhancement efforts or which driver education programs to pursue, in which areas and for which population segments.

Although this proposal does not solve the problem of sidewalks in poor condition and the evaluation has involved a very limited number of users, the positive results obtained encourage us to subsequently carry out other rigorous and systematic evaluations of both the usability and the impact of SmartMoving. Based on the preliminary results obtained, it is hoped that SmartMoving will help users to reduce the difficulty of moving around in Asunción.

\section{Conclusions and Future work}

In this paper we have presented the design and development process of the SmartMoving solution, a mobile application that seeks to promote citizen participation by trying to involve pedestrians to identify the best paths to walk. As there is no database that contains information on the state of the sidewalks in Asunción, this solution will be the first to generate this data with the help of user input.

On the one hand, the UCD process that we have applied helped to design the solution. As can be seen in Figure 7 and Figure 11 there is a remarkable difference between the initial prototype and the first functional version, designed and developed in the third iteration, thanks to feedback from users involved in the participatory process. On the other hand, the opinions about SmartMoving that we obtained from the interviews carried out were positive, stating that it will be of great help to users. Furthermore, the results obtained in the usability evaluation of the first functional version of SmartMoving were also favorable.

We believe that these types of technology-mediated citizen participation initiatives are a promising option for better solutions closer to people's needs. However, it will take time and effort for this type of technology to be widely adopted in our society to offer good solutions to problems of common interest.

Based on the comments and suggestions that we have collected in the interviews and also taking into account the data that will be generated with the use of the application, some future work can be suggested. In order to promote greater inclusion, UCD processes can be planned and executed for other user profiles. They could be people who use bicycles, tourists, people who have hearing or vision problems. According to the profile chosen, new needs can be identified, to later define and implement new functionalities. Services could also be implemented that help municipalities to manage complaints about irregularities in the sidewalks and streets, as well as to have a more updated and global vision of the situation. To try to reduce the risk of generating false data, areas to create reports could be verified, so that reports could be created only on the sidewalks, and not, for example, in areas of buildings or houses. Finally, to encourage the use of applications such as SmartMoving, which involve the active participation of users in detecting problems and therefore being able to propose solutions, it has been very useful to have incentive methods, not so much economic but rather recognition; and interesting ways of incentivising people could include options such as gamification [15]). 


\section{Acknowledgment}

This work was co-financed by Consejo Nacional de Ciencia y Tecnología (CONACYT) with resources from FEEI, within the framework of the project "SmartTraffic: sistemas colectivos adaptativos para una ciudad inteligente" (PINV15-166).

\section{Referencias}

[1] S. Hosio, J. Goncalves, V. Kostakos, and J. Riekki, "Crowdsourcing Public Opinion Using Urban Pervasive Technologies: Lessons From Real-Life Experiments in Oulu," Policy 6 Internet, vol. 7, no. 2, pp. 203-222, 2015. [Online]. Available: https://onlinelibrary.wiley.com/doi/abs/10.1002/poi3.90

[2] E. Graeff, "Crowdsourcing as Reflective Political Practice: Building a Location-Based Tool for Civic Learning and Engagement," Internet, Politics, and Policy, 2014. [Online]. Available: https://ssrn.com/abstract=2524967

[3] Secretaría Nacional de Tecnologías de la Información y Comunicación, "Encuesta sobre acceso y uso de Internet en Paraguay," http://gestordocumental.senatics.gov.py/share/s/ ntjnuNLeT8u3gbAHC6WeVw, 2017.

[4] M. Garreta Domingo and E. Mor Pera, Diseño centrado en el usuario. Universitat Oberta de Catalunya, 2010.

[5] M. F. Burian, P. Fauvety, N. Aquino, M. González, D. Romero, L. Cernuzzi, J. Paniagua, and R. Chenu-Abente, "Design of SmartMoving, an Application for Pedestrians with Reduced Mobility," in XLVI Latin American Computing Conference, CLEI 2020, Loja, Ecuador, October 19-23, 2020. IEEE, 2020, pp. 367-376. [Online]. Available: https://doi.org/10.1109/CLEI52000.2020.00049

[6] M. Fatecha, P. Fauvety, N. Aquino, M. González, D. Romero, L. Cernuzzi, J. Paniagua, and R. Chenu-Abente, "SmartMoving: una aplicación para peatones con movilidad reducida," in Proceedings of the XXIII Iberoamerican Conference on Software Engineering, CIbSE 2020, Curitiba, Paraná, Brazil, November 9-13, 2020, C. P. Ayala, L. Murta, D. S. Cruzes, E. Figueiredo, C. Silva, J. L. de la Vara, B. de França, M. Solari, G. H. Travassos, and I. Machado, Eds. Curran Associates, 2020, pp. 606-613. [Online]. Available: http://cibse2020.ppgia.pucpr.br/images/artigos/16/S16_P4.pdf

[7] S. Mirri, C. Prandi, and P. Salomoni, "Personalizing Pedestrian Accessible way-finding with mPASS," in 13th IEEE Annual Consumer Communications \& Networking Conference, CCNC 2016, Las Vegas, NV, USA, January 9-12, 2016. IEEE, 2016, pp. 1119-1124. [Online]. Available: https://doi.org/10.1109/CCNC.2016.7444946

[8] C. Prandi, P. Salomoni, and S. Mirri, "mPASS: Integrating people sensing and crowdsourcing to map urban accessibility," in 2014 IEEE 11th Consumer Communications and Networking Conference (CCNC), Jan 2014, pp. 591-595. [Online]. Available: https://doi.org/10.1109/CCNC.2014.6940491

[9] Q. Wang, B. Guo, L. Wang, T. Xin, H. Du, H. Chen, and Z. Yu, "CrowdWatch: Dynamic Sidewalk Obstacle Detection Using Mobile Crowd Sensing," IEEE Internet Things J., vol. 4, no. 6, pp. 2159-2171, 2017. [Online]. Available: https://doi.org/10.1109/JIOT.2017.2750324

[10] J. Cortellazzi, L. Foschini, C. R. D. Rolt, A. Corradi, C. A. A. Neto, and G. D. Alperstedt, "Crowdsensing and proximity services for impaired mobility," in IEEE Symposium on Computers and Communication, ISCC 2016, Messina, Italy, June 27-30, 2016. IEEE Computer Society, 2016, pp. 44-49. [Online]. Available: https://doi.org/10.1109/ISCC.2016.7543712

[11] P. Fauvety, M. Fatecha, A. Lugo, J. Olivera, N. Aquino, R. Chenú-Abente, J. Paniagua, and J. Saldívar, "Percepción ciudadana acerca de los problemas de movilidad de Asunción," Universidad Católica "Nuestra Señora de la Asunción", Tech. Rep., June 2018, https://drive.google.com/file/d/ 1le_-Tt-N-RaizqCKOR3m6LyOPfDdLc0v/view.

[12] J. Preece, H. Sharp, and Y. Rogers, Interaction Design: Beyond Human-Computer Interaction, 4th Edition. Wiley, 2015. [Online]. Available: http://www.id-book.com/fourthedition/index.php

[13] J. R. Lewis, "IBM computer usability satisfaction questionnaires: Psychometric evaluation and instructions for use," International Journal of Human-Computer Interaction, vol. 7, no. 1, pp. 57-78, 1995. [Online]. Available: https://doi.org/10.1080/10447319509526110 
[14] J. Lewis, "Measuring Perceived Usability: The CSUQ, SUS, and UMUX," International Journal of Human-Computer Interaction, vol. 34, no. 12, pp. 1148-1156, 2018. [Online]. Available: https://doi.org/10.1080/10447318.2017.1418805

[15] K. Huotari and J. Hamari, "Defining gamification: a service marketing perspective," in International Conference on Media of the Future, Academic MindTrek '12, Tampere, Finland, October 3-5, 2012, A. Lugmayr, Ed. ACM, 2012, pp. 17-22. [Online]. Available: https://doi.org/10.1145/2393132.2393137 